Bangladesh Journal of Neuroscience 2013; Vol. 29 (1) : 64-69

\title{
Juvenile Myasthenia Gravis: A Case Report and Review of Literatures
}

\author{
NARAYAN CHANDRA KUNDU ${ }^{1}$, MOUSHUMI SEN ${ }^{2}$, K.M. NAZMUL ISLAM JOY $^{3}$ \\ FEROJ AHMED QURAISH ${ }^{4}$
}

\begin{abstract}
:
Juvenile myasthenia gravis (JMG) is a rare autoimmune disorder of childhood. Pediatric presentation of MG is more common in Oriental than in Caucasian populations. JMG need to be differentiated from congenital myasthenia gravis which do not have haan autoimmune basis. An 11 years old girl presented with drooping of eye lids which was more marked at the later part of day and was gradually progressive. She had complained of double vision. She had no family history of myasthenia gravis. Ice pack test, repetitive nerve stimulation test, and anti acetylcholine receptor antibody test support the diagnosis. She was treated with pyridostigmine and was started as $30 \mathrm{mg}$ four times daily and increased to $60 \mathrm{mg} / \mathrm{qds}$. Subsequently her symptoms improved gradually and she became stable.
\end{abstract}

Key word: Juvenile myasthenia gravis (JMG). myasthenia gravis (MG)

Abbreviation: JMG(Juvenile myasthenia gravis). MG (myasthenia gravis ), NMJ (neuromuscular junction ), CMG (congenital MG), AchRA (Acetylcholine receptor antibody), MuSK (muscle specific kinase)

\section{Introduction:}

Myasthenia Gravis (MG) is an autoimmune disease in which antibodies are directed at the postsynaptic membrane of the neuromuscular junction (NMJ), leading to varying degrees of muscle weakness and fatigability. Where MG presents before 19 years of age, it is termed juvenile myasthenia gravis (JMG) ${ }^{1}$. JMG is a rare disorder of childhood and has many clinical features that are distinct from adult MG. Pediatric presentation of MG is more common in Oriental than in Caucasian populations ${ }^{2}$. Up to $50 \%$ of all cases of MG in Chinese populations present in childhood, mostly with ocular features, with a peak age at presentation of $5-10$ years ${ }^{3}$. MG occurs as one of three subtypes; transient neonatal, congenital, or juvenile MG. One half to $2 / 3$ of these children are not diagnosed within the first year of disease onset ${ }^{4}$. Autoimmune antibodies are directed against the postsynaptic membrane of the neuromuscular junction, resulting in muscle weakness and fatigability. Prepubertal children in particular have a higher prevalence of isolated ocular symptoms, lower frequency of acetylcholine receptor antibodies, and a higher probability of achieving remission. Diagnosis in young children can be complicated by the need to differentiate from congenital myasthenic syndromes, which do not have an autoimmune basis. Treatment commonly includes anticholinesterases, corticosteroids with or without steroid-sparing agents, and newer immune modulating agents. Plasma exchange and intravenous immunoglobulin (IVIG) are effective in preparation for surgery and in treatment of myasthenic crisis. Thymectomy increases remission rates. Diagnosis and management of children with JMG should take account of their developmental needs, natural history of the condition, and side-effect profiles of treatment options.

1. Associate Professor, Department of Neurology, Sir Salimullah Medial College \& Mitford Hospital, Dhaka.

2. Assistant Professor, Department of Biochemistry, Anwer Khan Modern Medical College, Dhanmondi, Dhaka.

3. Assistant Register, Department of Neurology, Sir Salimullah Medial College Mitford Hospital, Dhaka.

4. Professor of interventional neurology, National institute of neurosciences and hospital, Dhaka. 


\section{Case report:}

Miss Ishrat Jahan, 11 years old girl with nonconsanguineous parents, presented with the complaints of asymmetric dropping of upper eyelid for two months. Drooping was less apparent in the morning and worsened at the later part of day. She had also complaint of double vision on looking to lateral side. On enquiry she mentioned of difficulties in chewing food at the later part of intake. She had no nasal regurgitation and did not complain of any significant weakness while using limbs above the shoulder, getting up from the squatting position or climbing stairs. She had not complained of muscle pain or tenderness, skin rash or joint pain. On general examination no deviation from normal is noted. On neurological examination incomplete ptosis was noted, more on the right. Range of eye movement was normal. Pupil size was normal and equal on both sides and reacting normally to light. Examination of fundus and lower cranial nerves was found to be normal. Motor, sensory and cerebellar examination also revealed no abnormality. Bedside ice pack test was done and the test was found to be significant (on the right palpabral fissure width was increased about $6 \mathrm{~mm}$ and on the left it was about $5 \mathrm{~mm}$ ).

Complete blood count with ESR, CxR (PA view), chest CT, thyroid profile and ANF were normal. Repetitive nerve stimulation showed 25\% decrement of response at deltoid, $16 \%$ in ADM, $42 \%$ in orbicularis occuli (Fig-I, 2). The test is positive for post-synaptic neuromuscular junction disease. Anti-acetylcholine receptor antibody test report was highly positive $(26.30$, positive $>0.4)$. She was diagnosed as a case of juvenile myasthenia gravis. Pyridostigmine was started as $30 \mathrm{mg}$ four times daily and increased to $60 \mathrm{mg} /$ qds. Subsequently her symptoms improved gradually and she became stable.

$2011 / 10 / 01 u_{-}$

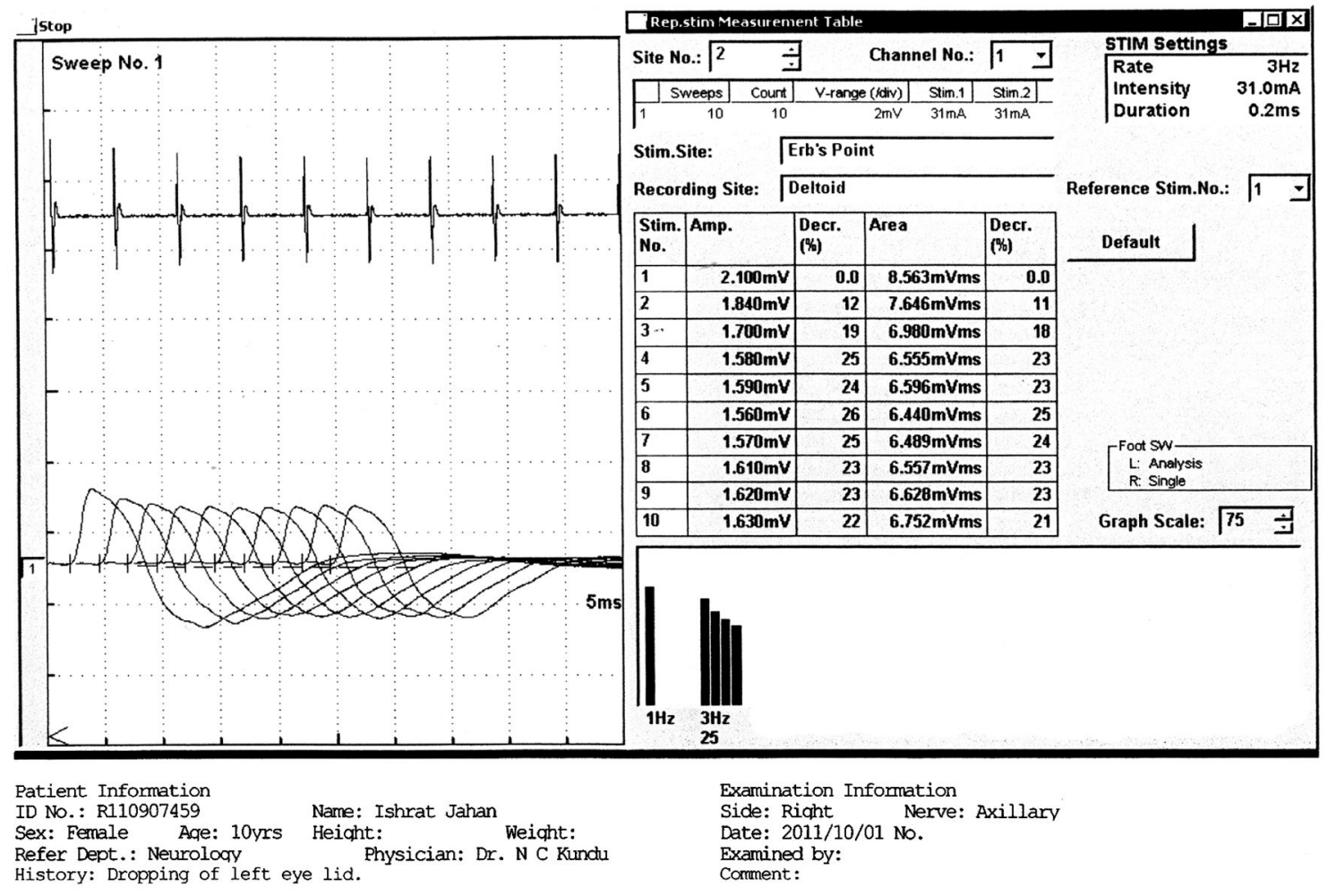

Fig-1: Repetitive nerve stimulation at Erb's point (recording site deltoid) 


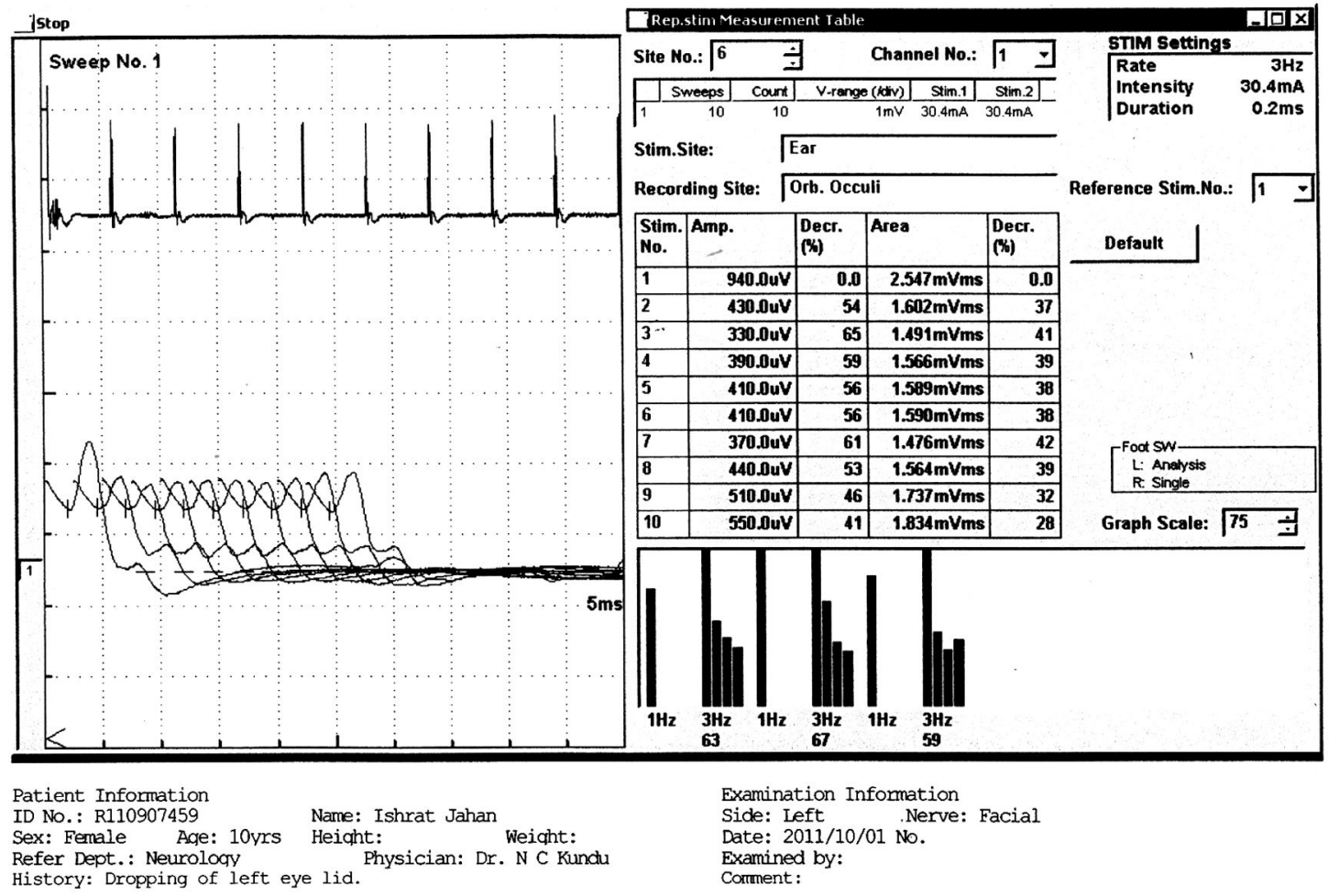

Fig-2: Repetitive nerve stimulation at Ear (recording site orbicularis Occuli)

\section{Discussion:}

Transient neonatal MG usually occurs in newborns, born to mothers with MG due to passive transmission of abnormal antibodies through the placenta ${ }^{5}$. This disorder differs from the rare cases of congenital MG (CMG), in which children have episodic apena and weakness of extra ocular, pharyngeal and respiratory muscles ${ }^{6}$. They inherit defects to the neuromuscular synapse, so they rarely have elevated levels of AchRA ${ }^{5}$.

JMG is the most common type of pediatric MG and the present case has exhibited this subtype. It is similar to the adult autoimmune disorder, but children often exhibit more severe symptoms. Prepubertal children presenting with JMG have some interesting and distinct clinical features compared with those developed JMG around or after puberty ${ }^{7-8}$. Prepubertal JMG is more likely to manifest as ocular myasthenia ${ }^{9}$. There is an equal male to female ratio, in contrast to the female predominance seen in peri or postpubertal children.
It also shows better prognosis, with a higher rate of spontaneous remission ${ }^{7-10}$. Peri or postpubertal patients presenting with JMG share more similarities with adult onset MG.

JMG is primarily diagnoses by clinical features. A number of diagnostic tools are available to aid diagnosis. An antibody to AchR supports the diagnosis of JMG. In this patient AchR antibody was done and showed high titer. Children who are negative for AchR antibodies can lead to difficulty in differentiating from CMG. Variable percentages (0-49\%) of MG patients without AchR antibodies are found to have antibodies against another neuromuscular junction protein, the muscle specific kinase (MuSK) ${ }^{11}$. MuSK positive MG is rare in children and represents a distinct subgroup of JMG, more severe disease with prominent facial and bulbar weakness and frequent respiratory crises ${ }^{12}$. Electrophysiology testing is invaluable in investigations of suspected JMG. Repetitive nerve stimulation will show a decrement in compound 
muscle action potential of $>10 \%$ by the $4^{\text {th }}$ or $5^{\text {th }}$ stimulation. Single fiber EMG (SFEMG) is especially useful in the diagnosis of seronegative MG and congenital myasthenic syndromes. Sensitivity for neuromuscular disorders is $97 \%$ and thus a normal result makes a diagnosis of myasthenia very unlikely ${ }^{13-14}$. In this reported case RNS was done and revealed 25\% decremented response at deltoid, $16 \%$ in ADM and $42 \%$ in orbicularis occuli muscle.

Although thymoma in children is rare, the thymus must be imaged once JMG has been diagnosed. Thymic hyperplasia is the commonest abnormality of the thymus in JMG ${ }^{15}$.

But X-ray chest and CT scan of chest of this patient revealed no abnormality.

Management of children with JMG should be delivered by a multidisciplinary team. Treatment has largely been extrapolated from adult studies and experience with adult patients. Side effect profiles and considerations are not always directly comparable between adult and pediatric population.

Acetylcholinesterase inhibitors are the first line treatment in JMG and provide symptomatic relief. Pyridostigmine is commonly used and is tailored to effects. Cautious use in MuSK positive children is advised because of risk of acetylcholine hypersensitivity ${ }^{16}$. Immunosuppression and immunomodulation is required to improve symptoms of JMG in most patients. Corticosteroids are effective and are the mainstay of therapy, can worsen symptoms if started at high doses ${ }^{17}$. Because of numerous adverse effects associated with long term use of steroids, steroids are often used in combination with steroid sparing agents. Azathioprine has been found to be effective. It can be used singly or in combination with steroid. Beneficial effects may take months to be seen ${ }^{18}$. Patients unresponsive or intolerant to azathioprine should be considered for other immunosuppressive agents including cyclosporin or cyclophosphamide 19-20. A Cochrane review suggests that cyclosporine either as monotherapy or with corticosteroids, or cyclophosphamides in conjunction with steroids improve symptoms of MG within 1 year ${ }^{21}$. A recent retrospective study which includes children as well concluded that Mycophenolate mofetil (MMF) when used as monotherapy or in conjunction with steroids is effective. Maximum effects may not be seen until after one year of treatment ${ }^{22}$. Tacrolimus has shown early and sustained improvement of symptoms in MG, allowing dose reduction of prednisolne and in many cases its complete withdrawal. These steroid sparing effects were seen within 6 months ${ }^{23-24}$. Rituximab has been used in refractory JMG 25.

Recent reviews of children including prepubertal patients, suggested increased remission rates after thymectomy. Caution needs to be taken in early childhood due to subsequent immunosuppression and the high rates of spontaneous remission in prepubertal presenters ${ }^{26-27}$. Current evidence suggests that thymectomy should not be recommended in MuSk positive disease as it is unclear whether it confers any benefit $28-29$. Thymectomy in pure OMG is controversial. Thymectomy is not proven to reduce risk of progression of OMG to generalized JMG and is not routinely indicated in pure OMG in children but has been performed in refractory cases ${ }^{30}$.

\section{Outcome:}

Outcomes in JMG have improved significantly over last decades, with better recognition, diagnosis, and more effective therapies, and long term prognosis is good ${ }^{31}$. Children with JMG exhibit higher rates of remission than adults. This includes spontaneous remission and remission following a period of drug therapy. Prepubertal children have the highest rates of spontaneous remission.

\section{Summary:}

JMG is a rare, autoimmune disorder of childhood that share many characteristics with the adult form of the disease. However, as described, there are many important aspects that are specific to the pediatric population. So, diagnosis and management of children with JMG should take account of their developmental needs, natural history of the condition, and side-effect profiles of treatment options. 


\section{References:}

1. Finnis MF, Jayawant S. Juvenile Myasthenia Gravis: APaediatric Perspective. Autoimmune diseases. Volume 2011, Article ID 404101.

2. Chiu H.C., Vincent A., Newsom-Davis J., Hsieh K.H., Hung T.P. Myasthenia gravis: population differences in disease expression and acetylcholine receptor antibody titres between Chinese and Caucasians. Neurology 1987; 37 (12); 1854-57.

3. Zhang $X$, Yang J, Xu J, Clinical and serological study of myasthenia gravis in HuBei Province, China. Journal of Neurology, Neurosurgery and Psychiatry 2007;78 (4): 386-90.

4. Cirillo ML. Neuromuscular emergencies. Clin Pediatr Emerg Med. 2008; 9: 88-95.

5. Sarat HB. Disorder of Neuromuscular Transmission and Motor Neurons. In: Kliegman RM., Behrman RE., Jenson HB, Stanton BF. Nelson Textbook of Pediatrics. $18^{\text {th }}$ ed. Philadelphia, PA: Saunders; 2007: 611.

6. Vincent A, Palace J, Hilton-Jones D. Myasthenia gravis. Lancet 2001; 357: 2122.

7. Andrews P.I., Massey JM., Howard JF., Sanders DB. Race, sex, and puberty influence onset, severity, and outcome in juvenile myasthenia gravis. Neurology 1994; 44 (7): 1208-14.

8. Evoli A, Batocchi AP., Bartoccioni E., Lino MM., Minisci C., Tonali P. Juvenile myasthenia gravis with prepubertal onset. Neuromuscular disorders 1996; 6 (2): 133-36.

9. Haliloglu G, Anlar B, Aysun S. Gender prevalence in childhood multiple sclerosis and myasthenia gravis. Journal of Child Neurology $2002 ; 17$ (5):390-2.

10. Chiang LM, Darras BT, Kang PB. Juvenile myasthenia gravis. Muscle and Nerve 2009. 39 (4): 423-31.

11. Vincent A, Leite MI. neuromuscular junction autoimmune disease: muscle specific kinase antibodies and treatment for myasthenia gravis. Current opinion in neurology 2005:18 (5): 519-25.
12. Pasnoor M, Wolfe GI, Nations S. Clinical findings in MuSK-antibody positive myasthenia gravis: a US experience. Muscle and Nerve 2010 ;41 (3): 370-4.

13. Pitt M. Neurophysiological strategies for the diagnosis of disorders of the neuromuscular junction in children. Developmental Medicine and Child Neurology 2008; 50 (5): 328-33.

14. Pitt M. Pediatric electromyography in the modern world: a personal view. Developmental Medicine and Child Neurology 2011; 53 (2): 120-24.

15. Linder A, Schalke B, Toyka KV. Outcome in Juvenile onset myasthenia gravis: a retrospective study with long term follow up of 79 patients. Journal of Neurology 1997; 244 (8): 515-20.

16. Punga A, Flink $\mathrm{R}$, Askmark $\mathrm{H}$, and Stalberg EV. Cholinergic neuromuscular hyperactivity in patients with myasthenia gravis seropositive for MuSK antibody. Muscle and Nerve 2006; 34 (1): 111-15.

17. Schneider-Gold C., Gajdos P., Toyka KV. and Hohlfeld RR. Corticosteroids for myasthenia gravis. Cochrane Database of Systemic Reviews 2006; 18 (2): 2828.

18. Palace J, Newsom-Davis J, and Lecky B. A randomized double blind trail of prednisolone alone or with azathioprine in myasthenia gravis. Neurology 1998;50 (6): 1778-83.

19. Tindal RSA, Rollins JA, Phillips JT, Greenlee RG, Wells L, and Belendiuk G. Preliminary results of a double-blind, randomized, placebocontrolled trail of cyclosporine in myasthenia gravis. The New England Journal of Medicine 1987;316 (12): 719-24.

20. de Feo LG, Schttlender J, Martelli NA, Molfino NA. Use of intravenous pulsed cyclophosphamide in severe, generalized myasthenia gravis. Muscle and Nerve 2002; 26 (1): 31-6.

21. Hart IK, Sathasivan S, and Sharshar T. Immunosuppressive agents for myasthenia gravis. Cochrane Database of Systemic Reviews. 17 (4); 2007 : CD0055224. 
22. Hehir MK, Burns TM, Alpers J, Conaway MR, Sawa M, Sanders DB. Mycophenolate mofetil in AchR-antibody positive myasthenia gravis: outcomes in 102 patients. Muscle and Nerve 2010;31 (5): 593-8.

23. Furukawa $\mathrm{Y}$, Yoshikawa $\mathrm{H}$, Iwasa $\mathrm{K}$, and Yamada M. Clinical efficacy and cytokine network modulating effects of tacrolimus in myasthenia gravis. Journal of Neuroimmunology 2008; 195 (1-2): 108-15.

24. Ponseti JM, Gamez J, Azem J, Lopez-Cano, Vilallonga $\mathrm{R}$, and Armengol M. Tacrolimus for myasthenia gravis: a clinical study of 212 patients. Annals of the New York Academy of Sciences 2008; 1132: 254-63.

25. Wylam ME, Anderson PM, Kuntz NL, and Rodriguez $V$. Successful treatment of refractory myasthenia gravis using rituximab: a pediatric case report. Journal of pediatrics 2004;143 (5): 674-7.

26. Hennessey IAM, Long A, Hughes I, and Humphrey G. Thymectomy for inducing remission in juvenile myasthenia gravis.
Pediatric surgery international 2011; 27 (6): 591-4.

27. Rodriguez M, Gomez MR, Howard FM, Taylor WF. Myasthenia gravis in children: long term follow up. Annals of neurology 1983; 13 (5): 504-10.

28. Sanders DB, El-Salem K, Massey JM, McConville J, and Vincent A. Clinical aspects of MuSK antibody positive seronegative MG. Neurology 2003; 60 (12): 1978-80.

29. Evoli A, Tonali PA, Padua L. clinical correlates with anti-MuSK antibodies in generalized seroneagtive myasthenia gravis. Brain 2003; 126 (10): 2301-11.

30. Kawaguchi N, Kuwabara Y, Nemoto Y. Treatment and outcome of myasthenia gravis: retrospective multi-center analysis of 470 Japanese patients, 1999-2000. Journal of the Neurological Science 2004; 224 (1): 43-7.

31. Grob D, Burnner N, Namba T, and Pagala M. Lifetime course of myasthenia gravis. Muscle and Nerve 2008; 37 (2): 141-9. 